\title{
P15: Führungskräfte - Kompetenzen, Aufgaben, Förderung
}

\author{
Angela Günther • Tonio Schönfelder
}

Online publiziert: 23. Oktober 2013

(C) Springer-Verlag Wien 2013

Einleitung/Fragestellung: Der Dienstleistungsbereich Gesundheit ist einer der am schnellsten wachsenden Märkte in Deutschland. Prognosen deuten an, dass bis 2030 bis zu 200.000 neue Arbeitsplätze entstehen werden. Hiermit ist ein entsprechender Bedarf an geeigneten Führungskräften verbunden. Die Zielstellung dieser Untersuchung war es deshalb, Anforderungen an Führungskräfte (Geschäftsführung, Pflegedirektion, Heimleitung) im Gesundheitswesen zu erfassen.

Methodik/Stichprobe: Es wurden 490 Stellenanzeigen von Fachzeitschriften (z. B. Krankenhausumschau, Das Krankenhaus), Jobbörsen (z. B. Monster), überregionalen Tageszeitungen (z. B. FAZ, Welt) sowie einer Totalerhebung von 1889 deutschen Krankenhäusern analysiert. Zusätzlich wurde ein elektronischer Fragebogen erstellt und per E-Mail an 900 zufällig ausgewählte Einrichtungen im Gesundheitswesen (100 Gesundheitsämter, 145 Krankenkassen, 313 Krankenhäuser und 342 Pflegeheime) versendet. Die Teilnehmer wurden anonym zu erforderlichen Hardskills und bevorzugten Softskills in Bezug auf eine leitende Führungskraft mit Personal- und Budgetverantwortung befragt. Die Rücklaufquote betrug $12 \%(N=106)$.
Ergebnisse: Circa 76\% der potentiellen Arbeitgeber erwarten Berufserfahrung, um in einer führenden Position tätig zu werden. Am häufigsten im Fragebogen genannt wurden 1-2 Jahre und mehr als 5 Jahre. Der Großteil der Arbeitgeber verlangt ein abgeschlossenes Studium (56 von 78 Befragten). Bevorzugt wird ein Bachelorabschluss oder ein Diplom. Schwerpunktmäßig wird ein Abschluss im Bereich der Wirtschaftswissenschaften (37\%), Gesundheitsmanagement (27\%) und Pflegemanagement (10\%) erwartet. Führungskräfte im Gesundheitswesen können mit jährlichen Bruttogehältern zwischen 40.000-60.000 € rechnen. Die wichtigsten Softskills waren Kommunikations- und Teamfähigkeit, Verantwortungsbewusstsein, Belastbarkeit, selbstständige Arbeitsweise sowie unternehmerisches Denken.

Diskussion/Schlussfolgerungen: Die Ergebnisse spiegeln einen steigenden Bedarf an qualifizierten Führungskräften im Gesundheitswesen wider. Potentielle Arbeitgeber erwarten überwiegend ein abgeschlossenes Studium im Bereich Wirtschaftswissenschaften und Gesundheitsmanagement als Voraussetzung für erfolgreiche Führungskräfte.

A. Günther · T. Schönfelder

Fakultät Gesundheits- und Pflegewissenschaften,

Westsächsische Hochschule Zwickau, Zwickau, Deutschland 